\title{
Clinical Feature and Outcome of Childhood Status Epilepticus in a Teaching Hospital, Odisha, India
}

Kedarnath Das ${ }^{1}$, Santosh K. Das ${ }^{1}$, Sarbeswar Pradhan ${ }^{2}$, Priyadarshini I. Sahoo ${ }^{3}$, Nirmal K. Mohakud ${ }^{2}$, Arakhita Swain $^{1}$, Saroj Satpathy ${ }^{1}$

1. Pediatrics, Srirama Chandra Bhanja Medical College and Hospital, Cuttack, IND 2. Pediatrics, Kalinga Institute of Medical Sciences, Bhubaneswar, IND 3. Medicine, Srirama Chandra Bhanja Medical College and Hospital, Cuttack, IND

Corresponding author: Nirmal K. Mohakud, nirmal.mahakud@kims.ac.in

\section{Abstract \\ Objectives}

The main aim is to find out the clinical feature and outcome of status epilepticus (SE) in children managed in a teaching hospital. The secondary aim is to identify the risk factors influencing the adverse outcomes.

\section{Methods}

In this prospective cohort, children aged 1 month to 14 years with SE as per the International League Against Epilepsy's new guideline (2016) who presented to the emergency department during the period of November 2017 to October 2019 were enrolled. Clinical profile, treatment, and outcome of cases $(n=94)$ were noted.

\section{Results}

The majority of children, 60 (63.82\%), were less than five years of age. Prior history of seizures was present in 33 (35.1\%) cases, whereas 61 (64.9\%) cases presented with SE as the first episode of seizure. In 14 (42.4\%) previous seizure cases, SE was due to drug default. No response to first-line antiepileptic drug (AED) was seen in 84 (89.37\%) cases. Acute symptomatic etiology was the commonest etiology of SE in 64 (68\%) cases, of which neuro-infections accounted for $44(46.80 \%)$ cases. Longer duration ( $>60$ minutes) of status ( $p$ $<0.01)$, ventilator support $(\mathrm{p}<0.0001)$, and circulatory impairment $(\mathrm{p}<0.0001)$ were attributable risk factors for mortality. A total of 28 children died (mortality rate, 29.8\%), and 11 showed the persistence of their neuro-deficit.

\section{Conclusions}

Neuro-infection is the most common etiology of SE in children. Longer duration of SE, more lag time for receiving the first AED, respiratory failure, and presence of shock are independent predictors for poor outcome. Hence, cessation of convulsion at the earliest leads to improved outcomes.

Review began 10/07/2020 Review ended 10/07/2020 Published 10/13/2020

\section{() Copyright 2020}

Das et al. This is an open access article distributed under the terms of the Creative Commons Attribution License CC-BY 4.0., which permits unrestricted use, distribution, and reproduction in any medium, provided the original author and source are credited.
Categories: Emergency Medicine, Neurology, Pediatrics

Keywords: convulsion, seizure, morbidity and mortality, antiepileptic drugs, status epilepticus, etiology

\section{Introduction}

Status epilepticus (SE) is a medical emergency, and its neurological outcome is a concern to every pediatrician in developing countries. The estimated convulsive status epilepticus (CSE) prevalence is 14.5 per 100,000 per year in developed countries [1], but population-based studies are not available from developing countries. There is significant morbidity (28-34\%) and mortality (7-22\%) with SE despite advancement in treatment protocols in the last decade [2,3]. It is documented that if convulsion persisted beyond 10 minutes, it leads to irreparable brain damage and difficulty in controlling the seizure [4]. Childhood survivors of SE may develop long-term consequences such as developmental delay, cognitive impairment, and recurrent seizure. Therefore, it is of paramount importance to control seizure activity within 5-10 minutes to prevent neuronal damage. Benzodiazepines (BZD) are the first-line drugs to control seizure within 5-10 minutes of convulsion [5,6]. It should be followed by second-line drugs in the next 10-20 minutes. If the convulsion does not get control, third-line drugs such as anesthetic medicines through continuous infusion should be given within 30-60 minutes [6].

Previous studies have described the clinical manifestation and outcomes of SE taking 30 minutes as a cutoff seizure duration [2,7]. Presently, SE is defined by the International League Against Epilepsy (ILAE) as any active seizure of $\geqslant 5$ minutes' duration or recurrent episodes of seizures without gaining consciousness in between the seizure episodes [8]. Using this definition, Chetan et al. [3] provided important information regarding etiological profile, response to various antiepileptic drugs (AEDs), and immediate outcome of SE in a prospective study from India. But data regarding clinical profile, predictors of morbidity, and mortality 
The objective of the study is to determine the clinical features, etiology, response to treatment with BZD, and immediate outcome, and to identify the risk factors influencing adverse outcomes in children with SE admitted to the pediatric ward/intensive care unit in a referral teaching hospital.

\section{Materials And Methods}

This prospective observational study included children between 1 month and 14 years of age admitted in the pediatrics ward of S. C. B. Medical College situated in a tertiary care teaching hospital from Eastern India over a two-year period (November 2017 to October 2019). Approval of the Institutional Ethical Committee was obtained before initiation of the study. During this period, 94 children with SE as per ILAE's new guideline (2016) [8] were admitted. SE was defined as active seizures of $\geqslant 5$ minutes' duration or recurrent episodes of seizures without gaining consciousness in between the seizure episodes [5]. Informed consent was obtained from parents or guardians of the included children. The total duration of seizure was obtained from the patient's mother or guardian, and other records of the referring doctor. Once the child was stabilized, data regarding age, sex, duration of seizures before and after admission, type and number of AEDs used for control, history of previous seizure pattern, adherence to treatment, perinatal history, developmental history, family history, and history of coexisting medical conditions were recorded. A general physical and detailed neurological examination was performed. Investigations, such as complete blood count, serum calcium, random blood sugar, serum sodium, urea and creatinine, neuroimaging, CSF (cerebrospinal fluid) examination, and IgM (immunoglobulin M) enzyme-linked immunosorbent assay for scrub typhus, were performed. Electroencephalography (EEG) was performed to ascertain etiology and guide treatment. During the hospital stay, recurrence of seizures, the subsequent need for intubation and mechanical ventilation, and days spent in the pediatric ICU were recorded. Detailed treatment, response, and outcome were also noted.

Type and etiology of SE were classified based on the SE classification report of the ILAE Task Force [8]. Refractory status epilepticus (RSE) was defined as the persistence of seizures in spite of using two appropriate anticonvulsants at therapeutic doses, with a minimum duration of status of 60 minutes (with observation and history). Though febrile seizures are a part of acute symptomatic etiology, it has been considered separately for analysis to avoid dilution of the severity of acute neurological insults.

Data were analyzed using SPSS Version 21 (IBM Corp., Armonk, NY, USA). A p-value of less than 0.05 was considered statistically significant. Furthermore, univariate analysis was used to find out the significance of various parameters in the outcome of the present study.

\section{Results}

Of the total 94 children studied, the median (IOR) age was 3 (1-7) years. The majority of children 60 (63.82\%) were less than five years of age. There was a male predominance (61 [64.9\%]). Out of hospital seizure onset was present in $82(87.2 \%)$ cases. A total of 49 (59.7\%) patients received pre-hospital treatment with diazepam (30) and phenytoin/phenobarbitone (19). Fever was present in 63 (67\%) cases before the onset of seizures. Prior history of seizures was present in 33 (35.1\%) cases, whereas the first episode of seizure with 61 (64.9\%) cases. Most of the children, 46 (48.9\%), had an SE duration of $<30$ minutes. The seizure was terminated after administration of BZD in 10 (10.63\%) cases, but second- and/or third-line drugs were needed in 84 (89.37\%) cases. However, 27 (28.72\%) patients progressed to RSE. Generalized seizures, mostly generalized tonic-clonic (GTC) seizures, were observed in 87 (92.6\%) children. Acute symptomatic etiology was the most common cause of SE in 64 (68\%) cases. Neuro-infections accounted for 44 (46.80\%) cases and febrile seizures for 5 (5.3\%) cases. Again, acute symptomatic etiologies were the major causes of SE in children less than five years of age, whereas remote symptomatic causes were more in children aged 3-10 years (Table 1). 


\section{Cureus}

\begin{tabular}{|c|c|}
\hline Characteristics & No $(\%)$ \\
\hline Median age, in years & $3(1-14)$ \\
\hline Less than 5 years & $60(63.82)$ \\
\hline Male & $61(64.9)$ \\
\hline History of fever before the onset & $63(67)$ \\
\hline Patients received pre-hospital treatment & 49 (59.7) \\
\hline First episode of seizure & $61(64.9)$ \\
\hline Prior history of seizures & $33(35.1)$ \\
\hline \multicolumn{2}{|l|}{ Duration of SE } \\
\hline$<30$ minutes & 46 (48.9) \\
\hline $30-60$ minutes & $4(4.3)$ \\
\hline$>60$ minutes & $44(46.8)$ \\
\hline \multicolumn{2}{|l|}{ Seizure type } \\
\hline Focal & $7(7.4)$ \\
\hline Generalized & $87(92.6)$ \\
\hline \multicolumn{2}{|l|}{ Etiology } \\
\hline Acute symptomatic & $64(68 \%)$ \\
\hline Viral encephalitis & $24(25.5)$ \\
\hline Pyogenic meningitis & $14(14.9)$ \\
\hline Tubercular meningitis & $3(3.2)$ \\
\hline Metabolic & $7(7.4)$ \\
\hline Hypertensive encephalopathy & $4(4.3)$ \\
\hline Intra-cranial hemorrhage & $2(2.1)$ \\
\hline Febrile seizure & $5(5.3)$ \\
\hline Scrub encephalitis & $3(3.2)$ \\
\hline Remote symptomatic & $17(18.1)$ \\
\hline Cerebral palsy & $12(12.8)$ \\
\hline Post-encephalitis hydrocephalus & $5(5.3)$ \\
\hline Progressive & $1(1.1)$ \\
\hline Cryptogenic & $12(12.8)$ \\
\hline
\end{tabular}

TABLE 1: Clinical and etiological features of SE children $(n=94)$

SE, status epilepticus

As shown in Table 2, the overall mortality was 28 (29.78\%). The acute symptomatic group had the highest mortality of 20 (71.42\%). Neurological deficit persisted in 11 (11.70\%) cases. 


\section{Cureus}

\begin{tabular}{|c|c|c|c|c|}
\hline Etiology & No. of cases & Death & Morbidity & Recovery \\
\hline Acute symptomatic & $64(68)$ & $20(71.42)$ & $8(72.72)$ & $36(65.45)$ \\
\hline Viral encephalitis & 24 & 8 & 1 & 15 \\
\hline Pyogenic meningitis & 14 & 5 & 0 & 9 \\
\hline Tubercular meningitis & 3 & 1 & 2 & 0 \\
\hline Metabolic & 7 & 3 & 1 & 3 \\
\hline Hypertensive encephalopathy & 4 & 2 & 1 & 1 \\
\hline Intra-cranial hemorrhage & 2 & 0 & 1 & 1 \\
\hline FIRES & 1 & 0 & 1 & 0 \\
\hline Stroke & 1 & 0 & 1 & 0 \\
\hline Febrile seizure & 5 & 1 & 0 & 4 \\
\hline Scrub encephalitis & 3 & 0 & 0 & 3 \\
\hline Remote symptomatic & $17(18.1 \%)$ & $5(29.41)$ & $1(9.09 \%)$ & $11(20 \%)$ \\
\hline Cerebral palsy and post-encephalitis sequelae & 12 & 2 & 1 & 9 \\
\hline Hydrocephalus & 5 & 3 & 0 & 2 \\
\hline Progressive neurological disease & $1(1.1 \%)$ & $1(100)$ & 0 & 0 \\
\hline Cryptogenic & 12 (12.76\%) & $2(16.67)$ & 2 (18.18\%) & 8 (14.54\%) \\
\hline Total & $94(100 \%)$ & 28 (29.78) & $11(11.70 \%)$ & $55(58.51 \%)$ \\
\hline
\end{tabular}

\section{TABLE 2: Treatment and outcome of status epilepticus in children}

Morbidity: after treatment of SE, patients who developed focal neurological deficits and neuro-cognitive impairment

FIRES, Febrile Infection-Related Epilepsy Syndrome

On analyzing the major contributing risk factors for mortality, duration of CSE > 60 minutes had higher mortality ( $\mathrm{n}=21$ [80.8\%]) compared to CSE $<30$ minutes $(\mathrm{n}=3[5.1 \%])(\mathrm{p}<0.01)$.The fatality in children with RSE was $85.2 \%$ compared to non-RSE 7.5\% ( $<$ 0.0001). Ventilator support $(p=0.0001)$ and circulatory impairment ( $\mathrm{p}=0.0001)$ were the important attributable risk factors for mortality (Table 3 ).

\begin{tabular}{|c|c|c|c|c|}
\hline Risk factor & Mortality (\%) & No mortality (\%) & $\square^{2}$ value & $\mathrm{p}$-Value \\
\hline \multicolumn{5}{|l|}{ Age } \\
\hline$<1$ year & $7(24.1)$ & 22 (75.9) & \multirow{4}{*}{4.83} & \multirow{4}{*}{0.18} \\
\hline $1-5$ years & $8(25.8)$ & $23(74.2)$ & & \\
\hline $5-10$ years & $6(26.1)$ & $17(73.9)$ & & \\
\hline$>10$ years & $7(63.6)$ & $4(36.4)$ & & \\
\hline \multicolumn{5}{|l|}{ Seizure episode } \\
\hline First episode & $19(31.1)$ & $42(68.9)$ & \multirow{2}{*}{0.15} & \multirow{2}{*}{0.69} \\
\hline Prior history of seizure & $9(27.3)$ & $24(72.7)$ & & \\
\hline \multicolumn{5}{|l|}{ Seizure types } \\
\hline Secondary generalized & $2(28.6)$ & $5(71.4)$ & \multirow[b]{3}{*}{1.80} & \multirow{3}{*}{0.61} \\
\hline Generalized clonic & $0(0)$ & $1(100)$ & & \\
\hline & & & & \\
\hline
\end{tabular}




\section{Cureus}

\begin{tabular}{|c|c|c|c|c|}
\hline Generalized tonic & $0(0)$ & $6(100)$ & & \\
\hline GTCS & $26(32.5)$ & $54(67.5)$ & & \\
\hline \multicolumn{5}{|l|}{ Lag time for receiving first AED } \\
\hline$<30$ minutes & $3(5.1)$ & $56(94.9)$ & \multirow{3}{*}{46.33} & \multirow{3}{*}{0.0001} \\
\hline $30-60$ minutes & $4(44.4)$ & $5(55.6)$ & & \\
\hline$>60$ minutes & $21(80.8)$ & $5(19.2)$ & & \\
\hline \multicolumn{5}{|l|}{ Response to treatment } \\
\hline RSE & $23(85.2)$ & $4(14.8)$ & \multirow{2}{*}{55.58} & \multirow{2}{*}{0.0001} \\
\hline NRSE & $5(7.5)$ & $62(92.5)$ & & \\
\hline \multicolumn{5}{|l|}{ Total duration of CSE } \\
\hline$<30$ minutes & $1(2.2)$ & $45(97.8)$ & \multirow{3}{*}{29.69} & \multirow{3}{*}{$<0.01$} \\
\hline $30-60$ minutes & $2(50.0)$ & $2(50.0)$ & & \\
\hline$>60$ minutes & $25(56.8)$ & $19(43.2)$ & & \\
\hline \multicolumn{5}{|l|}{ Pre-existing neurological deficit } \\
\hline Present & $7(35.0)$ & $13(65.0)$ & \multirow{3}{*}{0.33} & \multirow{2}{*}{0.56} \\
\hline Absent & $21(28.4)$ & $53(71.6)$ & & \\
\hline \multicolumn{4}{|l|}{ Ventilator } & \\
\hline Yes & $22(88.0)$ & $3(12.0)$ & \multirow{2}{*}{55.18} & \multirow{2}{*}{0.000} \\
\hline No & $6(8.7)$ & $63(91.3)$ & & \\
\hline \multicolumn{5}{|l|}{ Shock } \\
\hline Yes & $16(76.2)$ & $5(23.8)$ & \multirow{2}{*}{27.84} & \multirow{2}{*}{0.0001} \\
\hline No & $12(16.4)$ & $61(83.6)$ & & \\
\hline \multicolumn{5}{|l|}{ Etiology } \\
\hline Acute symptomatic (except febrile seizure) & $19(32.2)$ & $40(67.8)$ & \multirow{2}{*}{0.44} & \multirow{2}{*}{0.506} \\
\hline Other etiology & $9(25.7)$ & $26(74.3)$ & & \\
\hline
\end{tabular}

\section{TABLE 3: Analysis of risk factors for mortality in children with status epilepticus}

GTCS, generalized tonic-clonic seizure; AED, antiepileptic drug; RSE, resistant status epilepticus; NRSE, non-resistant status epilepticus; CSE, convulsive status epilepticus

\section{Discussion}

This prospective study elucidates the clinical outcome and response to treatment in childhood SE in a tertiary care teaching institute of a developing country. Taking into account the ILAE 2016 guideline, it is found that acute symptomatic etiology, mostly neuro-infection, is the most common cause of not only CSE but also increased relative risk for RSE, unlike existing literature [9,10]. More common diseases such as cerebral malaria, dengue infection of the central nervous system (CNS), and typhoid encephalopathy are hardly responsible for SE in Odisha compared to Africa [11].

In this study, it was found that children less than five years of age comprised the majority of the cases (63.8\%). Other studies have also found a higher prevalence in the younger age group [12,13]. As the first episode of convulsion has been theorized to be due to the underdeveloped mechanisms for control of seizure activity, there is a disruption of neuronal function with minimal abnormalities in younger children [1]. Also, younger age is more vulnerable to acute etiologies including febrile seizures. Of the acute symptomatic etiology, febrile seizures and cerebrospinal-vascular disease are the predominant cause of SE in developed countries [1,14], whereas in developing countries, CNS infection accounts for $28-47 \%$ of the etiological spectrum $[15,16]$. Acute symptomatic etiology (other than febrile seizures) was found to be the commonest etiology of SE in children younger than five years, and remote symptomatic etiology was common in 
children aged 3-10 years. This may be because of the high proportion of children with a history of prior seizures in the older age group.

A significantly high incidence of RSE with acute symptomatic etiology was found in the present study. Risk factors for RSE are sepsis, shock at presentation, pre-existing neurological abnormalities, and lag time for receiving the first AED. Children with RSE had a significantly prolonged length of stay in hospital. In this study, a less number $(n=11[11.70 \%])$ of patients had residual morbidity such as focal neurological deficits and neurocognitive impairment compared to another study $[17,18]$.

A requirement of mechanical ventilation was relatively higher in the present study because most patients referred to our hospital were in critical condition and refractory status. A similar finding was reported in a study from south India [17]. The common indications for putting on the ventilator were Glasgow Coma Scale (GCS) < 7 followed by refractory shock and respiratory failure. The median (IQR) hospital stay was 7 (58) days, with a range of 4 to 20 days. Need for prolonged stay might be due to admission to ICU and longer time taken for neurological recovery. Similar durations of stay were reported for other diseases previously $[19,20]$.

The mortality rate in the present study was similar to other Indian studies [21,22], whereas studies from developed countries reported comparatively less mortality (9-24\%) [23,24]. The risk factors for mortality in the present study included longer duration of SE, acute symptomatic etiology, delay in receiving first AED, RSE, the requirement of ventilator support, and circulatory impairment. Transportation from longer distance led to considerable delay in starting treatment and onset of SE. We have found that acute causes of SE trend toward the worse outcome. Duration of SE is the only potentially modifiable determinant of mortality [21]. Therefore, besides improvisation of peripheral institutes in critical care, need for high-end ambulances to transport children with SE should be the futuristic goal of the government.

\section{Limitations}

Few causes, such as the inborn error of metabolism, autoimmune encephalitis, specific and viral encephalitis, could not be specified due to lack of investigations.

\section{Conclusions}

Acute symptomatic etiology is the commonest cause of SE, predominantly with neuro-infections. Longer duration of SE, more lag time for receiving first AED, RSE, ventilator requirement, and presence of shock are independent predictors for poor outcomes. Upgradation of intensive care facilities even in district headquarter hospitals might improve outcomes of SE.

\section{Additional Information \\ Disclosures}

Human subjects: Consent was obtained by all participants in this study. S.C.B. Medical College IEC issued approval Appln. No-40. Animal subjects: All authors have confirmed that this study did not involve animal subjects or tissue. Conflicts of interest: In compliance with the ICMJE uniform disclosure form, all authors declare the following: Payment/services info: All authors have declared that no financial support was received from any organization for the submitted work. Financial relationships: All authors have declared that they have no financial relationships at present or within the previous three years with any organizations that might have an interest in the submitted work. Other relationships: All authors have declared that there are no other relationships or activities that could appear to have influenced the submitted work.

\section{Acknowledgements}

We are thankful to the Department of Pediatrics for providing the platform to conduct the study. We pay our gratitude to the statistician for her help in the analysis of the data. We are obliged to Dr. Mirabai Das for her help in the grammatical correction.

\section{References}

1. Chin RF, Neville BG, Peckham C, Bedford H, Wade A, Scott RC: Incidence, cause, and short-term outcome of convulsive status epilepticus in childhood: prospective population-based study. Lancet. 2006, 368:222-9. 10.1016/S0140-6736(06)69043-0

2. Sculier C, Gaínza-Lein M, Sánchez Fernández I, Loddenkemper T: Long-term outcomes of status epilepticus: a critical assessment. Epilepsia. 2018, 59:155-69. 10.1111/epi.14515

3. Chetan C, Sharma S, Mathur SB, Jain P, Aneja S: Clinical profile and short-term outcome of pediatric status epilepticus at a tertiary-care center in Northern India. Indian Pediatr. 2020, 57:213-7. 10.1007/s13312-0201753-6

4. Gaínzalein M, Fernández IS, Jackson M, et al.: Association of time to treatment with short-term outcomes for pediatric patients with refractory convulsive status epilepticus. JAMA. 2018, 74:410-18. 10.1001/jamaneurol.2017.4382 
5. Wilkes R, Tasker RC: Pediatric intensive care treatment of uncontrolled status epilepticus . Crit Care Clin. 2013, 29:239-57. 10.1016/j.ccc.2012.11.007

6. Glauser T, Shinnar S, Gloss D, et al.: Evidence-based guideline: treatment of convulsive status epilepticus in children and adults: report of the Guideline Committee of the American Epilepsy Society. Epilepsy Curr. 2016, 16:48-61. 10.5698/1535-7597-16.1.48

7. Smith DM, McGinnis EL, Walleigh DJ, Abend NS: Management of status epilepticus in children . J Clin Med. 2016, 5:47. 10.3390/jcm5040047

8. Trinka E, Cock H, Hesdorffer D, et al.: A definition and classification of status epilepticus - report of the ILAE Task Force on Classification of Status Epilepticus. Epilepsia. 2015, 56:1515-23. 10.1111/epi.13121

9. Tripathy SK, Mishra P, Dwibedi B, Priyadarshini L, Das RR: Clinico-epidemiological study of viral acute encephalitis syndrome cases and comparison to nonviral cases in children from Eastern India. J Glob Infect Dis. 2019, 11:7-12. 10.4103/igid.jgid_26_18

10. Kumar A, Mandal A, Sinha S, Singh A, Das RR: Prevalence, response to cysticidal therapy, and risk factors for persistent seizure in Indian children with neurocysticercosis. Int J Pediatr. 2017, 2017:8983958. $10.1155 / 2017 / 8983958$

11. Sadarangani M, Seaton C, Scott JA, et al.: Incidence and outcome of convulsive status epilepticus in Kenyan children: a cohort study. Lancet Neurol. 2008, 7:145-50. 10.1016/S1474-4422(07)70331-9

12. Gulati S, Kalra V, Sridhar MR: Status epilepticus in Indian children in a tertiary care center . Indian J Pediatr. 2005, 1:105-8. 10.1007/BF02760691

13. Kumar M, Kumari R, Narain NP: Clinical profile of status epilepticus (SE) in children in a tertiary care hospital in Bihar. J Clin Diagn Res. 2014, 8:14-7. 10.7860/JCDR/2014/9288.4579

14. Bergamo S, Parata F, Nosadini M, et al.: Children with convulsive epileptic seizures presenting to Padua pediatric emergency department: the first retrospective population-based descriptive study in an Italian health district. J Child Neurol. 2015, 30:289-95. 10.1177/0883073814538670

15. Madhu PK, Krithika R: Convulsive status epilepticus in children: clinical profile and outcome in a tertiary care hospital. Int J Contemp Pediatr. 2019, 6:280-7. 10.18203/2349-3291.ijcp20190093

16. Murthy JM, Jayalaxmi SS, Kanikannan MA: Convulsive status epilepticus: clinical profile in a developing country. Epilepsia. 2007, 48:2217-23. 10.1111/j.1528-1167.2007.01214.x

17. Lingappa L, Konanki R, Patel R, Vooturi S, Jayalakshmi S: Clinical profile and outcome of refractory convulsive status epilepticus in older children from a developing country. Seizure Eur J Epilepsy. 2016, 36:31-5. 10.1016/j.seizure.2016.01.014

18. Jain P, Sharma S, Dua T, Barbui C, Das RR, Aneja S: Efficacy and safety of anti-epileptic drugs in patients with active convulsive seizures when no IV access is available: systematic review and meta-analysis. Epilepsy Res. 2016, 122:47-55. 10.1016/j.eplepsyres.2016.02.006

19. Das P, Singh D, Mohakud NK, Nayak RK, Mohakud NK: Epidemiological and clinical features of scrub typhus in Odisha, Eastern India. Med J DY Patil Vidyapeeth. 2019, 2:419-23. 10.4103/mjdrdypu.mjdrdypu_236_18

20. Mohakud NK, Mishra M, Tripathy R, Mishra MR: Incidence and risk factors for prolonged stay in children hospitalised with pneumonia. J Clin Diagn Res. 2018, 12:12-4. 10.7860/JCDR/2018/35460.11944

21. Sadik KC, Mishra D, Juneja M, Jhamb U: Clinico-etiological profile of pediatric refractory status epilepticus at a public hospital in India. J Epilepsy Res. 2019, 9:36-41. 10.14581/jer.19004

22. Kalita J, Nair PP, Misra UK: A clinical, radiological and outcome study of status epilepticus from India . J Neurol. 2010, 257:224-29. 10.1007/s00415-009-5298-9

23. Ferlisi M, Hocker S, Trinka E, Shorvon S; International Steering Committee of the StEp Audit: Etiologies and characteristics of refractory status epilepticus cases in different areas of the world: results from a global audit. Epilepsia. 2018, 59:100-7. 10.1111/epi.14496

24. Kravljanac R, Jovic N, Djuric M, Jankovic B, Pekmezovic T: Outcome of status epilepticus in children treated in the intensive care unit: a study of 302 cases. Epilepsia. 2011, 52:358-63. 10.1111/j.15281167.2010.02943.x 